PROCEEDINGS OF THE

AMERICAN MATHEMATICAL SOCIETY

Volume 126, Number 12, December 1998, Pages 3481-3494

S 0002-9939(98)04575-4

\title{
PARTIAL ACTIONS OF GROUPS AND ACTIONS OF INVERSE SEMIGROUPS
}

\author{
RUY EXEL
}

(Communicated by Palle E. T. Jorgensen)

\begin{abstract}
Given a group $G$, we construct, in a canonical way, an inverse semigroup $\mathcal{S}(G)$ associated to $G$. The actions of $\mathcal{S}(G)$ are shown to be in one-to-one correspondence with the partial actions of $G$, both in the case of actions on a set, and that of actions as operators on a Hilbert space. In other words, $G$ and $\mathcal{S}(G)$ have the same representation theory.

We show that $\mathcal{S}(G)$ governs the subsemigroup of all closed linear subspaces of a $G$-graded $C^{*}$-algebra, generated by the grading subspaces. In the special case of finite groups, the maximum number of such subspaces is computed.

A "partial" version of the group $C^{*}$-algebra of a discrete group is introduced. While the usual group $C^{*}$-algebra of finite commutative groups forgets everything but the order of the group, we show that the partial group $C^{*}$-algebra of the two commutative groups of order four, namely $Z / 4 Z$ and $Z / 2 Z \oplus Z / 2 Z$, are not isomorphic.
\end{abstract}

\section{INTRODUCTION}

A semigroup $S$ is said to be an inverse semigroup provided there exists, for each $x$ in $S$, a unique element $x^{*}$ in $S$ such that

(i) $x x^{*} x=x$,

(ii) $x^{*} x x^{*}=x^{*}$.

According to one of the earliest results on this subject, the Vagner-Preston representation Theorem [7, V.1.10], every inverse semigroup is isomorphic to a subsemigroup of $\mathcal{I}(X)$, the inverse semigroup of partially defined bijective maps on a set $X$. The interested reader will also find in [7] the basic facts about inverse semigroups.

By a partially defined map on $X$ we mean a map $\phi: A \rightarrow B$, where $A$ and $B$ are subsets of $X$. Given such a $\phi$ we will denote by $\operatorname{dom}(\phi)$ and $\operatorname{ran}(\phi)$ its domain $A$ and range $B$, respectively. The multiplication rule on $\mathcal{I}(X)$ is given by composition of partial maps in the largest domain where it makes sense, that is, if $\phi$ and $\psi$ are elements of $\mathcal{I}(X)$, then $\operatorname{dom}(\phi \psi)=\psi^{-1}(\operatorname{ran}(\psi) \cap \operatorname{dom}(\phi))$ and $\operatorname{ran}(\phi \psi)=\phi(\operatorname{ran}(\psi) \cap \operatorname{dom}(\phi))$.

The representation theory of inverse semigroups studies homomorphisms (i.e. semigroup homomorphisms) $\pi: S \rightarrow \mathcal{I}(X)$ where $S$ is the inverse subgroup under

Received by the editors June 19, 1996 and, in revised form, April 16, 1997.

1991 Mathematics Subject Classification. Primary 20M18, 46L05, 20M30.

The author was partially supported by CNPq, Brazil.

(C)1998 American Mathematical Society 
scrutiny and $X$ is a set. Adopting the point of view of the theory of group actions, we make the following:

1.1. Definition. An action of the inverse semigroup $S$ on the set $X$ is a homomorphism $\pi: S \rightarrow \mathcal{I}(X)$.

In view of the recent interest in the concept of partial actions of groups on $C^{*}$ algebras (see [5], [9], [4], [12], [14], [11], [1]), it is interesting to study the more general concept of partial actions of groups on sets, as defined below.

1.2. Definition. Given a group $G$ and a set $X$, a partial action of $G$ on $X$ is a pair

$$
\Theta=\left(\left\{D_{t}\right\}_{t \in G},\left\{\theta_{t}\right\}_{t \in G}\right),
$$

where, for each $t$ in $G, D_{t}$ is a subset of $X$ and $\theta_{t}: D_{t^{-1}} \rightarrow D_{t}$ is a bijective map, satisfying, for all $r$ and $s$ in $G$

(i) $D_{e}=X$ and $\theta_{e}=i d_{X}$, the identity map on $X$ (here, as always, $e$ denotes the identity element of $G$ ),

(ii) $\theta_{r}\left(D_{r^{-1}} \cap D_{s}\right)=D_{r} \cap D_{r s}$,

(iii) $\theta_{r}\left(\theta_{s}(x)\right)=\theta_{r s}(x), \quad x \in D_{s^{-1}} \cap D_{s^{-1} r^{-1}}$.

Thus, a partial action of $G$ on $X$ is also a map from $G$ into $\mathcal{I}(X)$, just like the case for actions of inverse semigroups. However, the map $t \in G \mapsto \theta_{t} \in \mathcal{I}(X)$ does not satisfy $\theta_{r} \theta_{s}=\theta_{r s}$ as one might imagine, but rather we have $\theta_{r} \theta_{s} \subseteq \theta_{r s}$, that is, $\theta_{r s}$ is an extension of $\theta_{r} \theta_{s}$.

One of our major goals will be to introduce, for each group $G$, an inverse semigroup, which we will denote by $\mathcal{S}(G)$. This turns out to be a very natural object to consider, although we have found no account of it in the literature concerning semigroups. The interest in studying $\mathcal{S}(G)$ stems from the fact that the partial actions of $G$ on a set $X$ are in one-to-one correspondence with the homomorphisms from $\mathcal{S}(G)$ into $\mathcal{I}(X)$.

This problem was first considered in Sieben's [14] master thesis, where a partial answer was given in the case of actions on $C^{*}$-algebras. The inverse subgroup constructed there, however, is not intrinsically obtained from $G$, but it depends also on the partial action under consideration.

\section{The INVERSE SEMIGROUP ASSOCIATED TO A GROUP}

Throughout this section $G$ will denote a fixed group. The crucial property of partial actions which will enable us to construct our inverse semigroup is that, although $\theta_{r s}$ is only an extension of $\theta_{r} \theta_{s}$ (notation as in the previous section), we always have $\theta_{r} \theta_{s} \theta_{s^{-1}}=\theta_{r s} \theta_{s^{-1}}$. More will be said about this property later.

2.1. Definition. We let $\mathcal{S}(G)$ denote the universal semigroup defined via generators and relations as follows. To each element $t$ in $G$ we take a generator $[t]$ (from any fixed set having as many elements as $G$ ). For every pair of elements $t, s$ in $G$ we consider the relations

(i) $\left[s^{-1}\right][s][t]=\left[s^{-1}\right][s t]$,

(ii) $[s][t]\left[t^{-1}\right]=[s t]\left[t^{-1}\right]$,

(iii) $[s][e]=[s]$,

(iv) $[e][s]=[s]$. 
A rule of thumb to remember the first two relations is that $[s][t]$ can be replaced by $[s t]$ only if there is either an $\left[s^{-1}\right]$ hanging out on the left hand side, or a $\left[t^{-1}\right]$ on the right.

Note that, as a consequence of (i) and (iii), we have that $[t]\left[t^{-1}\right][t]=[t]$, which is the first hint that $\mathcal{S}(G)$ might be shown to be an inverse semigroup. This will, in fact, be accomplished below. We should also remark that axiom (iv) is a consequence of the previous ones, since we have, for each $s$ in $G$, that

$$
[e][s]=\left[s s^{-1}\right][s]=[s]\left[s^{-1}\right][s]=[s] .
$$

Therefore (iv) could be removed from the definition without any significant consequence.

By the universal property of semigroups defined via generators and relations we have:

2.2. Proposition. Given a semigroup $S$ and a map $f: G \rightarrow S$ satisfying

(i) $f\left(s^{-1}\right) f(s) f(t)=f\left(s^{-1}\right) f(s t)$,

(ii) $f(s) f(t) f\left(t^{-1}\right)=f(s t) f\left(t^{-1}\right)$,

(iii) $f(s) f(e)=f(s)$,

there exists a unique homomorphism $\tilde{f}: \mathcal{S}(G) \rightarrow S$ such that $\tilde{f}([t])=f(t)$.

The proof is elementary but we would like to point out that, according to the remarks above, the relation $f(e) f(s)=f(s)$, corresponding to (2.1.iv), need not be included as it is a consequence of (i) - (iii).

2.3. Proposition. There is an involutive anti-automorphism $*: \mathcal{S}(G) \rightarrow \mathcal{S}(G)$ such that, for every $t$ in $G,[t]^{*}=\left[t^{-1}\right]$.

Proof. Let $\mathcal{S}(G)^{o p}$ be the opposite semigroup, that is $\mathcal{S}(G)^{o p}$ coincides with $\mathcal{S}(G)$ in all respects, except that multiplication of the elements $\alpha$ and $\beta$ in $\mathcal{S}(G)^{o p}$ is defined by

$$
\alpha \bullet \beta:=\beta \alpha
$$

where $\beta \alpha$, on the right hand side, corresponds to the usual multiplication in $\mathcal{S}(G)$. Define $f: G \rightarrow \mathcal{S}(G)$ by $f(t)=\left[t^{-1}\right]$. It is easy to see that, for $s$ and $t$ in $G$ we have

(i) $f\left(s^{-1}\right) \bullet f(s) \bullet f(t)=f\left(s^{-1}\right) \bullet f(s t)$,

(ii) $f(s) \bullet f(t) \bullet f\left(t^{-1}\right)=f(s t) \bullet f\left(t^{-1}\right)$,

(iii) $f(s) \bullet f(e)=f(s)$.

So, by $(2.2), f$ extends to a homomorphism $*: \mathcal{S}(G) \rightarrow \mathcal{S}(G)^{o p}$, which, when viewed as a map from $\mathcal{S}(G)$ to itself, rather than into the opposite semigroup, is an antiautomorphism satisfying the desired properties.

An element $\varepsilon$ in a semigroup is said to be idempotent if $\varepsilon^{2}=\varepsilon$. Idempotents in $\mathcal{S}(G)$ are studied next.

2.4. Proposition. For every $t$ in $G$ let $\varepsilon_{t}=[t]\left[t^{-1}\right]$. Then, for each $t$ and $s$ in $G$,

(i) $\varepsilon_{t}$ is a self-adjoint idempotent in the sense that $\varepsilon_{t}^{*}=\varepsilon_{t}=\varepsilon_{t}^{2}$,

(ii) $[t] \varepsilon_{s}=\varepsilon_{t s}[t]$,

(iii) $\varepsilon_{t}$ and $\varepsilon_{s}$ commute.

Proof. The first statement is trivial. As for (ii), using (i) and (ii) of (2.1), as well as the fact that $[t]\left[t^{-1}\right][t]=[t]$, we have,

$$
[t] \varepsilon_{s}=[t][s]\left[s^{-1}\right]=[t s]\left[s^{-1}\right]=[t s]\left[s^{-1} t^{-1}\right][t s]\left[s^{-1}\right]=[t s]\left[s^{-1} t^{-1}\right][t]=\varepsilon_{t s}[t] .
$$


Finally, using (ii), we have

$$
\varepsilon_{t} \varepsilon_{s}=[t]\left[t^{-1}\right] \varepsilon_{s}=[t] \varepsilon_{t^{-1} s}\left[t^{-1}\right]=\varepsilon_{s}[t]\left[t^{-1}\right]=\varepsilon_{s} \varepsilon_{t} .
$$

2.5. Proposition. Every element $\alpha$ in $\mathcal{S}(G)$ admits a decomposition

$$
\alpha=\varepsilon_{r_{1}} \ldots \varepsilon_{r_{n}}[s],
$$

where $n \geq 0$ and $r_{1}, \ldots, r_{n}, s$ are elements of $G$. In addition, one can assume that

(i) $r_{i} \neq r_{j}$ for $i \neq j$,

(ii) $r_{i} \neq s$ and $r_{i} \neq e$ for all $i$.

Proof. Let $S$ be the subset of $\mathcal{S}(G)$ consisting of those $\alpha$ that do admit a decomposition as above. Since $n=0$ is allowed, we see that each $[s]$ belongs to $S$. To prove the statement it is then enough to verify that $S$ is a subsemigroup of $\mathcal{S}(G)$, in view of the fact that the $[s]$ 's form a generating system for $\mathcal{S}(G)$.

Let $\alpha=\varepsilon_{r_{1}} \ldots \varepsilon_{r_{n}}[s]$. It suffices to demonstrate that $\alpha[t]$ belongs to $S$, since this will prove $S$ to be a right ideal and hence a subsemigroup. Now, note that

$$
[s][t]=[s]\left[s^{-1}\right][s][t]=[s]\left[s^{-1}\right][s t]=\varepsilon_{s}[s t] .
$$

So

$$
\alpha[t]=\varepsilon_{r_{1}} \ldots \varepsilon_{r_{n}}[s][t]=\varepsilon_{r_{1}} \ldots \varepsilon_{r_{n}} \varepsilon_{s}[s t] \in S .
$$

With regard to the last part of the statement, since the idempotents $\varepsilon_{r_{j}}$ commute with each other, it is easy to see how to eliminate repetitions among the $\varepsilon_{r}$ 's, if any, as well as any occurrence of $\varepsilon_{e}$, which is the identity element of $\mathcal{S}(G)$.

Also, if some $r_{i}=s$, then $\varepsilon_{r_{i}}=[s]\left[s^{-1}\right]$ and, again by commutativity of idempotents, we have

$$
\alpha=\varepsilon_{r_{1}} \ldots \widehat{\varepsilon_{r_{i}}} \ldots \varepsilon_{r_{n}}[s]\left[s^{-1}\right][s]=\varepsilon_{r_{1}} \ldots \widehat{\varepsilon_{r_{i}}} \ldots \varepsilon_{r_{n}}[s] .
$$

So $\varepsilon_{r_{i}}$ can be eliminated.

2.6. Definition. If $\alpha$ is written as $\alpha=\varepsilon_{r_{1}} \ldots \varepsilon_{r_{n}}[s]$, in such a way that conditions (i) and (ii) of (2.5) are verified, we say that $\alpha$ is in standard form.

We now present some more evidence to support our earlier claim (to be proved below) that $\mathcal{S}(G)$ is an inverse semigroup.

2.7. Proposition. For each $\alpha$ in $\mathcal{S}(G)$ one has $\alpha \alpha^{*} \alpha=\alpha$ and $\alpha^{*} \alpha \alpha^{*}=\alpha^{*}$.

Proof. Let $\alpha$ be represented as $\alpha=\varepsilon_{r_{1}} \ldots \varepsilon_{r_{n}}[s]$. We then have

$$
\alpha \alpha^{*} \alpha=\varepsilon_{r_{1}} \ldots \varepsilon_{r_{n}}[s]\left[s^{-1}\right] \varepsilon_{r_{n}} \ldots \varepsilon_{r_{1}} \varepsilon_{r_{1}} \ldots \varepsilon_{r_{n}}[s]=\varepsilon_{r_{1}} \ldots \varepsilon_{r_{n}}[s]\left[s^{-1}\right][s]=\alpha .
$$

The second statement follows from the first one and our knowledge that $*$ is an anti-automorphism of $\mathcal{S}(G)$.

The only task remaining, before we can assert that $\mathcal{S}(G)$ is in fact an inverse semigroup, is to show that $\alpha^{*}$ is the unique element satisfying the identities in the statement of our previous result. However, uniqueness results in algebraic structures originated from generators and relations are difficult to establish, unless we can find a separating family of representations. This is the purpose of our next section. 


\section{Representations of $\mathcal{S}(G)$}

In this section we shall use the term "representation" in a very loose way, meaning any homomorphism of $\mathcal{S}(G)$ into a semigroup. These representations will often be obtained with the aid of (2.2).

The most obvious representation of $\mathcal{S}(G)$ one can think of is the map

$$
\partial: \mathcal{S}(G) \rightarrow G
$$

given by $\partial([t])=t$. We shall refer to $\partial(\alpha)$ as the degree of $\alpha$. Clearly $\partial\left(\varepsilon_{r_{1}} \ldots \varepsilon_{r_{n}}[s]\right)$ $=s$.

We next discuss a more subtle representation of $\mathcal{S}(G)$. Let $\mathcal{P}_{e}(G)$ be the set of all finite subsets of $G$ which contain the unit element $e$. Thus $G$ and $\{e\}$ are, respectively, the biggest and the smallest elements of $\mathcal{P}_{e}(G)$. The representation we are about to consider is a homomorphism from $\mathcal{S}(G)$ into the semigroup $\mathcal{F}\left(\mathcal{P}_{e}(G)\right)$ consisting of all functions from $\mathcal{P}_{e}(G)$ into itself, under the composition rule.

Thus, for each $t \in G$ let us denote by $\phi_{t}$ the map

$$
\phi_{t}: \mathcal{P}_{e}(G) \rightarrow \mathcal{P}_{e}(G)
$$

given by $\phi_{t}(E)=t E \cup\{e\}$, for each $E \in \mathcal{P}_{e}(G)$. Here, as usual, $t E$ stands for the set $\{t s: s \in E\}$.

Observe that, since $e \in E$, we can also write $\phi_{t}(E)=t E \cup\{t, e\}$.

3.1. Proposition. The map $t \in G \mapsto \phi_{t} \in \mathcal{F}\left(\mathcal{P}_{e}(G)\right)$ satisfies the properties (i) - (iii) of (2.2) and hence there is a unique representation $\Lambda: \mathcal{S}(G) \rightarrow \mathcal{F}\left(\mathcal{P}_{e}(G)\right.$ ) such that $\Lambda([t])=\phi_{t}$.

Proof. We prove only (2.2.i). For every $t, s \in G$ and each $E \in \mathcal{P}_{e}(G)$, we have

$$
\phi_{s^{-1}} \phi_{s} \phi_{t}(E)=\phi_{s^{-1}} \phi_{s}(t E \cup\{t, e\})=\phi_{s^{-1}}(s t E \cup\{s t, s, e\})=t E \cup\left\{t, e, s^{-1}\right\} \text {, }
$$

while

$$
\phi_{s^{-1}} \phi_{s t}(E)=\phi_{s^{-1}}(s t E \cup\{s t, e\})=t E \cup\left\{t, s^{-1}, e\right\} .
$$

Observe that, for any $r$ in $G$ and $E$ in $\mathcal{P}_{e}(G)$, one has $\Lambda\left(\varepsilon_{r}\right)(E)=E \cup\{r\}$. In particular, if $\alpha=\varepsilon_{r_{1}} \ldots \varepsilon_{r_{n}}[s]$, we can easily prove that $\Lambda(\alpha)$, when applied to the singleton $\{e\}$, gives $\left\{r_{1}, \ldots, r_{n}, s, e\right\}$.

Based on the existence of these two representations we may prove uniqueness of our decomposition.

3.2. Proposition. Every $\alpha$ in $\mathcal{S}(G)$ admits a unique standard decomposition

$$
\alpha=\varepsilon_{r_{1}} \ldots \varepsilon_{r_{n}}[s]
$$

up to the order of the $\varepsilon_{r}$ 's.

Proof. As observed above, $\Lambda(\alpha)(\{e\})=\left\{r_{1}, \ldots, r_{n}, s, e\right\}$ and also $\partial(\alpha)=s$. So, if one has another standard decomposition $\alpha=\varepsilon_{t_{1}} \ldots \varepsilon_{t_{m}}[u]$, then, on the one hand, we would have $s=u$ and, on the other, $\left\{r_{1}, \ldots, r_{n}, s, e\right\}=\left\{t_{1}, \ldots, t_{m}, u, e\right\}$. Hence

$$
\left\{r_{1}, \ldots, r_{n}, s, e\right\} \backslash\{s, e\}=\left\{t_{1}, \ldots, t_{m}, u, e\right\} \backslash\{u, e\}
$$

which implies that

$$
\left\{r_{1}, \ldots, r_{n}\right\}=\left\{t_{1}, \ldots, t_{m}\right\}
$$


With this we can compute the order of $\mathcal{S}(G)$ for finite groups.

3.3. Theorem. If $G$ is a finite group of order $p$, then $\mathcal{S}(G)$ has $2^{p-2}(p+1)$ elements.

Proof. In fact, there are $2^{p-1}$ elements in $\mathcal{S}(G)$ of the form $\varepsilon_{r_{1}} \ldots \varepsilon_{r_{n}}[e]$ and for $p-1$ possible choices of $s \neq e$, there are $2^{p-2}$ elements of the form $\varepsilon_{r_{1}} \ldots \varepsilon_{r_{n}}[s]$. The total number is then $2^{p-1}+(p-1) 2^{p-2}=2^{p-2}(p+1)$.

Therefore we see that the order of $\mathcal{S}(G)$ grows exponentially with the order of $G$. For a group of order 28 , for example, the order of $\mathcal{S}(G)$ is $1,946,157,056$. As for less dramatic examples, groups of order 2 through 10 cause the order of $\mathcal{S}(G)$ to be $3,8,20,48,112,256,576,1280$ and 2816 , respectively.

3.4. Theorem. For every group $G, \mathcal{S}(G)$ is an inverse semigroup.

Proof. Assume $\alpha \in \mathcal{S}(G)$ admits, in addition to $\alpha^{*}$, another "inverse", that is an element $\beta$ in $\mathcal{S}(G)$ such that $\alpha \beta \alpha=\alpha$ and $\beta \alpha \beta=\beta$. Write, in standard form,

$$
\alpha=\varepsilon_{r_{1}} \ldots \varepsilon_{r_{n}}[s]
$$

and

$$
\beta^{*}=\varepsilon_{t_{1}} \ldots \varepsilon_{t_{m}}[u] .
$$

Therefore $\beta=\left[u^{-1}\right] \varepsilon_{t_{1}} \ldots \varepsilon_{t_{m}}$ and we have $s=\partial(\alpha)=\partial(\alpha \beta \alpha)=s u^{-1} s$ from which it follows that $u=s$. We also have

$$
\alpha \beta \alpha=\varepsilon_{r_{1}} \ldots \varepsilon_{r_{n}}[s]\left[s^{-1}\right] \varepsilon_{t_{1}} \ldots \varepsilon_{t_{m}} \varepsilon_{r_{1}} \ldots \varepsilon_{r_{n}}[s]=\varepsilon_{r_{1}} \ldots \varepsilon_{r_{n}} \varepsilon_{t_{1}} \ldots \varepsilon_{t_{m}}[s]=\alpha .
$$

By the uniqueness of standard decompositions, we see that

$$
\left\{r_{1}, \ldots, r_{n}\right\} \cup\left\{t_{1}, \ldots, t_{m}\right\}=\left\{r_{1}, \ldots, r_{n}\right\}
$$

so

$$
\left\{t_{1}, \ldots, t_{m}\right\} \subseteq\left\{r_{1}, \ldots, r_{n}\right\} .
$$

The same argument, applied to the identity $\beta^{*} \alpha^{*} \beta^{*}=\beta^{*}$, yields

$$
\left\{r_{1}, \ldots, r_{n}\right\} \subseteq\left\{t_{1}, \ldots, t_{m}\right\},
$$

so we conclude that $\beta=\alpha^{*}$.

3.5. Remark. Here are some consequences of (3.2). Note that, if $e$ is an idempotent in $\mathcal{S}(G), x \in \mathcal{S}(G)$ and $x e$ is also idempotent, then $x$ must necessarily be an idempotent. This says that $\mathcal{S}(G)$ is an E-unitary semigroup (see [7, p. 55]). However $\mathcal{S}(G)$ is not a Clifford semigroup (see [7, IV.2]) since idempotents are not central by (2.4). We thank the referee for pointing out these facts.

\section{ACtions of INVERSE SEMIGRoups Vs. PARTIAL ACTIONS OF GROUPS}

Recall that a partial action of a group $G$ on a set $X$ is a map $\theta: G \rightarrow \mathcal{I}(X)$ satisfying the conditions of (1.2). Our next proposition is designed to rephrase these conditions in terms of the semigroup structure of $\mathcal{I}(X)$.

4.1. Proposition. Let $G$ be a group and $X$ be a set. $A$ map $\theta: G \rightarrow \mathcal{I}(X)$ gives a partial action of $G$ on $X$ if and only if, for all $s, t \in G$, we have

(i) $\theta_{s} \theta_{t} \theta_{t^{-1}}=\theta_{s t} \theta_{t^{-1}}$,

(ii) $\theta_{e}=i d_{X}$. 
In this case $\theta$ also satisfies

(iii) $\theta_{s^{-1}} \theta_{s} \theta_{t}=\theta_{s^{-1}} \theta_{s t}$.

Proof. Taking $s=t^{-1}$ in (i), we have

$$
\theta_{t^{-1}} \theta_{t} \theta_{t^{-1}}=\theta_{e} \theta_{t^{-1}}=\theta_{t^{-1}}
$$

Replacing the roles of $t$ and $t^{-1}$, we also have $\theta_{t} \theta_{t^{-1}} \theta_{t}=\theta_{t}$. So, by the uniqueness of inverses in inverse semigroups, we conclude that $\theta_{t}^{*}=\theta_{t^{-1}}$. Define $D_{t}=\operatorname{ran}\left(\theta_{t}\right)$; so the above conclusion tells us that

$$
\operatorname{dom}\left(\theta_{t}\right)=\operatorname{ran}\left(\theta_{t}^{*}\right)=\operatorname{ran}\left(\theta_{t^{-1}}\right)=D_{t^{-1}} .
$$

That is, $\theta_{t}$ is a map from $D_{t^{-1}}$ to $D_{t}$, as required. Now, for any $s, t \in G$ we have

$$
\theta_{t^{-1}} \theta_{s^{-1}}=\theta_{t^{-1}} \theta_{s^{-1}} \theta_{s} \theta_{s^{-1}}=\theta_{t^{-1} s^{-1}} \theta_{s} \theta_{s^{-1}} .
$$

So, in particular, the domains of these maps must agree. On the one hand we have

$$
\operatorname{dom}\left(\theta_{t^{-1}} \theta_{s^{-1}}\right)=\theta_{s}\left(D_{s^{-1}} \cap D_{t}\right) .
$$

Noting that $\theta_{s} \theta_{s^{-1}}$ is the identity function on $D_{s}$, we have, on the other hand,

$$
\operatorname{dom}\left(\theta_{t^{-1} s^{-1}} \theta_{s} \theta_{s^{-1}}\right)=D_{s} \cap D_{s t} .
$$

Now the third condition of (1.2) can easily be checked. The converse is left to the reader.

With respect to the last part of the statement note that, since $\theta_{t^{-1}} \theta_{s^{-1}} \theta_{s}=$ $\theta_{t^{-1} s^{-1}} \theta_{s}$ and $\theta_{t}^{*}=\theta_{t^{-1}}$, we have

$$
\theta_{s^{-1}} \theta_{s} \theta_{t}=\left(\theta_{t^{-1}} \theta_{s^{-1}} \theta_{s}\right)^{*}=\left(\theta_{t^{-1} s^{-1}} \theta_{s}\right)^{*}=\theta_{s^{-1}} \theta_{s t} \text {. }
$$

Our main result may now be stated.

4.2. Theorem. For every group $G$ and any set $X$, there is a one-to-one correspondence between

(a) partial actions of $G$ on $X$, and

(b) actions of $\mathcal{S}(G)$ on $X$.

Proof. By (2.2), homomorphisms from $\mathcal{S}(G)$ into $\mathcal{I}(X)$ are in one-to-one correspondence with maps from $G$ to $\mathcal{I}(X)$ satisfying (i)-(iii) of (2.2). As we have seen in (4.1), these correspond to partial actions of $G$ on $X$.

\section{Graded $C^{*}$-Algebras}

References for $C^{*}$-algebra theory are [10] and [6].

5.1. Definition. Let $G$ be a group. A $C^{*}$-algebra $A$ is said to be graded over $G$, or $G$-graded, if $A$ is equipped with a linearly independent family of closed linear subspaces $\left\{A_{t}\right\}_{t \in G}$, such that for all $s, t \in G$

(i) $A_{s} A_{t} \subseteq A_{s t}$,

(ii) $A_{t}^{*}=A_{t^{-1}}$,

(iii) $A$ is the closure of $\bigoplus_{t \in G} A_{t}$.

In this case, each $A_{t}$ is called a grading subspace of $A$. 
We would like to show that $\mathcal{S}(G)$ plays an important role in describing certain subspaces of $A$, related to its graduation. For example, given $s$ and $t$ in $G$, the product $A_{s} A_{t}$, although contained in $A_{s t}$, need not coincide with it, and, in fact, need not even be dense there. We could, therefore, ask what is the maximum number of subspaces of $A$ one can obtain by taking the closure of finite products of grading subspaces.

Before we proceed, let us agree on the following slightly unusual notational convention: If $X$ and $Y$ are subsets of a $C^{*}$-algebra, then $X Y$ will denote the closed linear span of the set of products $x y$ with $x \in X$ and $y \in Y$.

Returning to our graded algebra, let us denote $D_{t}=A_{t} A_{t^{-1}}$. It is easy to show that $A_{t}$ is a $D_{t^{-}} D_{t^{-1}}$-Hilbert bimodule. A good reference for the theory of Hilbert bimodules is [8]. As is the case for all right Hilbert modules, the product $A_{t} D_{t^{-1}}$ must coincide with $A_{t}[8,1.1 .4]$. This translates to $A_{t} A_{t^{-1}} A_{t}=A_{t}$.

5.2. Definition. Given a $C^{*}$-algebra $A$, let $\mathcal{P}_{\ell}(A)$ be the set of all closed linear subspaces of $A$, equipped with the semigroup structure under which the product of $X$ and $Y$ is defined to be the closed linear span of the set of products $x y$ with $x \in X$ and $y \in Y$, as mentioned earlier.

At this point one cannot oversee the fact that the map

$$
t \in G \mapsto A_{t} \in \mathcal{P}_{\ell}(A)
$$

provides a key connection between the theory of graded $C^{*}$-algebras and the theory of semigroups we have been discussing so far. As already mentioned, this map is not a homomorphism of semigroups but:

5.3. Proposition. Given a graded $C^{*}$-algebra $A=\overline{\bigoplus_{t \in G} A_{t}}$, then, for every $s$ and $t$ in $G$ one has

(i) $A_{s^{-1}} A_{s} A_{t}=A_{s^{-1}} A_{s t}$,

(ii) $A_{s} A_{t} A_{t^{-1}}=A_{s t} A_{t^{-1}}$,

(iii) $A_{s} A_{e}=A_{s}$.

Proof. Based on the fact that $A_{t} A_{t^{-1}} A_{t}=A_{t}$ and that $A_{s} A_{t} \subseteq A_{s t}$ we have

$$
A_{s^{-1}} A_{s} A_{t} \subseteq A_{s^{-1}} A_{s t}=A_{s^{-1}} A_{s} A_{s^{-1}} A_{s t} \subseteq A_{s^{-1}} A_{s} A_{t},
$$

which proves (i). The proof of (ii) is quite similar, while (iii) can be proved as follows:

$$
A_{s} A_{e} \subseteq A_{s}=A_{s} A_{s^{-1}} A_{s} \subseteq A_{s} A_{e} .
$$

5.4. Theorem. Given a graded $C^{*}$-algebra $A=\overline{\bigoplus_{t \in G} A_{t}}$, there is a correspondence which assigns, for each $\alpha$ in $\mathcal{S}(G)$, a closed subspace $A^{\alpha}$ of $A$ satisfying, for all $\alpha$ and $\beta$ in $\mathcal{S}(G)$ and all $t$ in $G$,

(i) $A^{[t]}=A_{t}$,

(ii) if $\partial(\alpha)=t$, then $A^{\alpha}$ is contained in $A_{t}$,

(iii) the closed linear span of the product of $A^{\alpha}$ by $A^{\beta}$ is exactly equal to $A^{\alpha \beta}$.

Proof. This is an immediate consequence of (5.3) and (2.2).

As a consequence, we see that the collection $\left\{A^{\alpha}\right\}_{\alpha \in \mathcal{S}(G)}$ is closed under multiplication, i.e., it is a subsemigroup of $\mathcal{P}_{\ell}(A)$. Since it contains the $A_{t}$ 's, we have provided an answer for the question posed after (5.1), concerning the maximum number of different subspaces of $A$ that one can obtain by taking finite products of 
grading subspaces. In the specific case of finite groups, that number is at most the order of $\mathcal{S}(G)$, which we have seen to equal $2^{p-2}(p+1)$, where $p$ is the order of $G$.

The question begging to be asked at this point is whether one can produce a $G$-graded $C^{*}$-algebra such that the $A^{\alpha}$ are all distinct. In fact this question makes sense even for infinite groups. The answer is yes but we are not yet prepared to provide an example right now. First we need to develop our theory a little further.

\section{Hilbert space Representations}

Contrary to our previous treatment of representations of semigroups, simply as homomorphisms into another semigroup, we shall now make a more systematic study of representations of inverse semigroups on Hilbert spaces. Therefore, let $H$ denote Hilbert's space and denote by $\mathcal{B}(H)$ the collection of all bounded linear operators on $H$.

6.1. Definition. Let $S$ be an inverse semigroup. A (Hilbert space) representation of $S$ on $H$ is a map $\pi: S \rightarrow \mathcal{B}(H)$ such that, for all $\alpha, \beta$ in $S$

(i) $\pi(\alpha \beta)=\pi(\alpha) \pi(\beta)$,

(ii) $\pi\left(\alpha^{*}\right)=\pi(\alpha)^{*}$.

If $S$ has a unit $e$, we shall also require that

(iii) $\pi(e)=I$.

Note that, since $\alpha \alpha^{*} \alpha=\alpha$, the same holds for $\pi(\alpha)$, which is then a partially isometric operator on $H$ for each $\alpha \in S$. Also, if $\varepsilon$ is an idempotent in $S$, one necessarily has $\varepsilon^{*}=\varepsilon$ and hence $\pi(\varepsilon)$ is a selfadjoint projection on $H$.

The related notion for groups is that of a partial representation:

6.2. Definition. Let $G$ be a group. A partial representation of $G$ on $H$ is a map $\pi: G \rightarrow \mathcal{B}(H)$ such that, for all $t, s \in G$,

(i) $\pi(s) \pi(t) \pi\left(t^{-1}\right)=\pi(s t) \pi\left(t^{-1}\right)$,

(ii) $\pi\left(t^{-1}\right)=\pi(t)^{*}$,

(iii) $\pi(e)=I$.

Note that (i)-(iii) imply that $\pi\left(s^{-1}\right) \pi(s) \pi(t)=\pi\left(s^{-1}\right) \pi(s t)$. So, regarding $\mathcal{B}(H)$ as a semigroup under multiplication (i.e., composition of operators), we see that a partial representation of $G$ satisfies the conditions of (2.2) and hence there exists a homomorphism

$$
\tilde{\pi}: \mathcal{S}(G) \rightarrow \mathcal{B}(H)
$$

such that $\tilde{\pi}([t])=\pi(t)$ for all $t$ in $G$. It is not hard to see that $\tilde{\pi}$ also satisfies $\tilde{\pi}\left(\alpha^{*}\right)=\tilde{\pi}(\alpha)^{*}$, and hence $\tilde{\pi}$ is a representation of $\mathcal{S}(G)$ on $H$ in the sense of (6.1).

Conversely, given a representation $\rho$ of $\mathcal{S}(G)$ on $H$, it is easy to see that the map

$$
t \in G \mapsto \rho([t]) \in \mathcal{B}(H)
$$

is a partial representation of $G$. We have therefore proven:

6.3. Proposition. There is a one-to-one correspondence between

(a) partial representations of $G$ on $H$, and

(b) representations of $\mathcal{S}(G)$ on $H$. 
One of the important features of the theory of $C^{*}$-algebras is that several different representation theories are governed by a $C^{*}$-algebra. The theory of partial representations of groups is no exception and we shall now construct a $C^{*}$-algebra, called the partial group $C^{*}$-algebra of $G$, which will play the corresponding role with respect to partial group representations.

The representation theory of inverse semigroups has received much attention and one can also associate Banach and $C^{*}$-algebras of interest to the theory. A key result, due to Barnes [2], states that the $l_{1}$ algebra of an inverse semigroup has a separating family of irreducible representations. Also, Duncan and Paterson [3] have studied both the reduced and the full $C^{*}$-algebras of an inverse semigroup.

However, given our interest in the special case of the inverse semigroup associated to a group, with all of its characteristic features, we shall prefer to develop our theory from scratch.

Let $G$ denote a group, which we will consider fixed for the remainder of this section.

An auxiliary $C^{*}$-algebra in our study is the universal $C^{*}$-algebra, denoted $A$, defined via generators and relations as follows. The set of generators consists of a symbol $P_{E}$ for each finite subset $E \subseteq G$, and the relations are:

(i) $P_{E}=P_{E}^{*}$,

(ii) $P_{E} P_{F}=P_{E \cup F}$,

for all possible choices of $E$ and $F$. Note that each $P_{E}$ will then be a projection and, if $\emptyset$ denotes the empty set, $P_{\emptyset}$ will serve as a unit for $A$. Obviously, $A$ is an abelian $C^{*}$-algebra, so by Gelfand's Theorem, $A=C(X)$ for some compact space $X$. For the time being the description of $A$ as a universal $C^{*}$-algebra will be quite appropriate for our purposes, so we shall postpone the study of its spectrum until needed.

Since the collection of all $P_{E}$ 's is closed under multiplication and adjoints, one has that $A$ is the closed linear span of these elements.

Let $A_{e}$ be the ideal of $A$ generated by $P_{\{e\}}$, that is, $A_{e}=P_{\{e\}} A$. As before, $A_{e}$ is the closed linear span of the set $\left\{P_{E}: e \in E\right\}$. Also $A_{e}$ is a unital $C^{*}$-algebra with unit $P_{\{e\}}$.

For each $t$ in $G$, let $\theta_{t}$ be the automorphism of $A$ specified by $\theta_{t}\left(P_{E}\right)=P_{t E}$. Observe that $A_{e}$ is not invariant under $\theta_{t}$, but if we let

$$
D_{t}=\overline{\operatorname{span}}\left\{P_{E}: e, t \in E\right\},
$$

then $D_{t}$ is an ideal in $A_{e}$ and the restriction of $\theta_{t}$ to $D_{t^{-1}}$ is a $C^{*}$-algebra isomorphism

$$
\theta_{t}: D_{t^{-1}} \rightarrow D_{t}
$$

which lives fully inside of $A_{e}$. This is an example of a partial automorphism of $A_{e}$, as defined in [5], and the map $t \mapsto \theta_{t}$ fits the definition of a partial action of $G$ on $A_{e}$ given in [4] and [9]. In particular, we can form the crossed product of $A_{e}$ by $G$.

6.4. Definition. The partial group $C^{*}$-algebra of $G$ is the $C^{*}$-algebra $C_{p}^{*}(G)$ given by the crossed product [4], [9] of $A_{e}$ by $\theta$, that is,

$$
C_{p}^{*}(G)=A_{e} \rtimes_{\theta} G \text {. }
$$

The following result improves upon (6.3) and shows the relevance of $C_{p}^{*}(G)$ in the theory of partial representations of $G$. 
6.5. Theorem. There is a one-to-one correspondence between

(a) partial representations of $G$ on $H$,

(b) representations of $\mathcal{S}(G)$ on $H$ and

(c) $C^{*}$-algebra representations of $C_{p}^{*}(G)$ on $H$.

Proof. Let $r, s$ be in $G$ and let $E$ and $F$ be finite subsets of $G$ containing $\{e, r\}$ and $\{e, s\}$, respectively. Then $P_{E}$ is in $D_{r}$ and $P_{F}$ is in $D_{s}$ and we have, following [4] and $[9]$,

$$
\left(P_{E} \delta_{r}\right) *\left(P_{F} \delta_{s}\right)=\theta_{r}\left(\theta_{r}^{-1}\left(P_{E}\right) P_{F}\right) \delta_{r s}=\theta_{r}\left(P_{r^{-1} E \cup F}\right) \delta_{r s}=P_{E \cup r F} \delta_{r s} .
$$

Consider, for each $t$ in $G$, the element $u_{t}=P_{\{e, t\}} \delta_{t} \in C_{p}^{*}(G)$. Based on the computation above, it is easy to verify that $u_{s} u_{t} u_{t^{-1}}=u_{s t} u_{t^{-1}}$, that $u_{t^{-1}}=u_{t}^{*}$ and that $u_{e}=1$. This said, it is easy to see that every representation of $C_{p}^{*}(G)$, when computed on the elements $u_{t}$, gives a partial representation of $G$.

Conversely, suppose we are given a representation $\pi$ of $\mathcal{S}(G)$ on $H$. Recall that the idempotents $\varepsilon_{r}$ in $\mathcal{S}(G)$ are defined by $\varepsilon_{r}=[r]\left[r^{-1}\right]$. For each finite set $E=\left\{r_{1}, \ldots, r_{n}\right\} \subseteq G$, define

$$
Q_{E}=\pi\left(\varepsilon_{r_{1}} \ldots \varepsilon_{r_{n}}\right) .
$$

This is a projection in $\mathcal{B}(H)$ and we have $Q_{E} Q_{F}=Q_{E \cup F}$ for all $E$ and $F$, so there exists a representation $\rho$ of $A$ on $H$ such that $\rho\left(P_{E}\right)=Q_{E}$. Also let $u_{t}=\pi([t])$. If $E$ contains $\left\{e, t^{-1}\right\}$, then by (2.4), we have

$$
\begin{aligned}
u_{t} \rho\left(P_{E}\right) u_{t^{-1}} & =\pi\left([t] \varepsilon_{r_{1}} \ldots \varepsilon_{r_{n}}\left[t^{-1}\right]\right)=\pi\left(\varepsilon_{t r_{1}} \ldots \varepsilon_{t r_{n}}[t]\left[t^{-1}\right]\right) \\
& =\pi\left(\varepsilon_{t r_{1}} \ldots \varepsilon_{t r_{n}}\right)=\rho\left(P_{t E}\right)=\rho\left(\theta_{t}\left(P_{E}\right)\right) .
\end{aligned}
$$

So we get a covariant representation of the partial action of $G$ on $A_{e}$, in the sense of $[9,2.8]$, and hence a representation $\rho \times u$ of $C_{p}^{*}(G)$. In particular

$$
\rho \times u\left(P_{\{e, t\}} \delta_{t}\right)=\rho\left(P_{\{e, t\}}\right) u_{t}=Q_{\{e, t\}} \pi([t])=\pi\left(\varepsilon_{e} \varepsilon_{t}[t]\right)=\pi([t]) .
$$

For our following result it will be important to understand the algebra $A$ more concretely. For this purpose let $K$ be the power set of $G$. Among many different descriptions for $K$, one can use the model

$$
K=\prod_{t \in G}\{0,1\} .
$$

Equipped with the product topology, $K$ becomes a compact Hausdorff space. When $G$ is an infinite countable group, for example, $K$ is homeomorphic to Cantor's set. For each $t$ in $G$ let $Q_{t} \in C(K)$ be the $t^{t h}$ coordinate function, i.e.,

$$
Q_{t}: x \in K \mapsto x_{t} \in \mathbf{C} .
$$

For each finite set $E \subseteq G$ let $Q_{E}=\prod_{t \in E} Q_{t}$. Clearly $Q_{E} Q_{F}=Q_{E \cup F}$ so we get a $C^{*}$-algebra homomorphism

$$
\Phi: A \rightarrow C(K)
$$

such that $\Phi\left(P_{E}\right)=Q_{E}$. 
6.6. Proposition. $A$ and $C(K)$ are isomorphic via the homomorphism $\Phi$ above.

Proof. Surjectivity is an immediate consequence of the Stone-Weierstrass Theorem. To prove injectivity let $a \in A$ be non-zero. Take a character $f$ of $A$ such that $f(a) \neq 0$. For each $t$ in $G$ observe that $P_{\{t\}}$ is a projection in $A$, so $f\left(P_{\{t\}}\right)$ is a complex number in $\{0,1\}$. Define $x$ in $K$ by

$$
x=\left(f\left(P_{\{t\}}\right)\right)_{t \in G} .
$$

For all $t$ we have $\Phi\left(P_{\{t\}}\right)(x)=Q_{t}(x)=f\left(P_{\{t\}}\right)$ so, observing that the $P_{\{t\}}$ generate $A$, we see that we must also have $\Phi(a)(x)=f(a) \neq 0$, from which we conclude that $\Phi(a) \neq 0$ and hence that $\Phi$ is injective.

It is well known that a crossed product by a partial action is a graded $C^{*}$-algebra, and hence so is $C_{p}^{*}(G)$. This is also the case for the full (as opposed to partial) group $C^{*}$-algebra $C^{*}(G)$ (see [10], for definitions). However the grading of $C^{*}(G)$ is rather uninteresting from our point of view since it is saturated, that is, the product of the grading subspaces $A_{t}$ and $A_{s}$ always coincides with $A_{t s}$. In terms of the notation of (5.4), this simply says that $A^{\alpha}=A_{\partial(\alpha)}$. The situation for the partial group algebra is a lot more interesting.

6.7. Theorem. Let $C_{p}^{*}(G)=\overline{\bigoplus_{t \in G} B_{t}}$ be the G-grading of $C_{p}^{*}(G)$ arising from its construction as a partial crossed product. Also let

$$
\alpha \in \mathcal{S}(G) \mapsto B^{\alpha} \in \mathcal{P}_{\ell}(A)
$$

be the semigroup homomorphism referred to in (5.4). Then, if $\alpha \neq \beta$, one has $B^{\alpha} \neq B^{\beta}$.

Proof. We initially claim that if $\alpha=\varepsilon_{r_{1}} \ldots \varepsilon_{r_{n}}[s] \in \mathcal{S}(G)$, then

$$
B^{\alpha}=\overline{\operatorname{span}}\left\{P_{E} \delta_{s}: e, r_{1}, \ldots, r_{n}, s \in E\right\} .
$$

In fact, if $n=0$, then $B^{\alpha}=B^{[s]}=B_{s}$ and the result follows from the definition of $B_{s}$ as $B_{s}=D_{s} \delta_{s}$ and the fact that $D_{s}$ is spanned by the $P_{E}$ 's with $e, s \in E$.

Let $n>0$ and $\gamma=\varepsilon_{r_{2}} \ldots \varepsilon_{r_{n}}[s]$ so $\alpha=\varepsilon_{r_{1}} \gamma$ and $B^{\alpha}=B^{\left[r_{1}\right]\left[r_{1}^{-1}\right] \gamma}=B_{r_{1}} B_{r_{1}^{-1}} B^{\gamma}$. By induction we may assume that $B^{\gamma}=\overline{\operatorname{span}}\left\{P_{E} \delta_{s}: e, r_{2}, \ldots, r_{n}, s \in E\right\}$.

On the other hand, it is easy to see that $B_{r_{1}} B_{r_{1}^{-1}}=D_{r_{1}}=\overline{\operatorname{span}}\left\{P_{F}: e, r_{1} \in F\right\}$. We thus have

$$
B_{\alpha}=\overline{\operatorname{span}}\left\{P_{F} P_{E} \delta_{s}: e, r_{1} \in F ; e, r_{2}, \ldots, r_{n}, s \in E\right\} .
$$

Observing that $P_{F} P_{E}=P_{F \cup E}$ we see that the claim is proved.

Returning to the proof of our theorem, suppose

$$
\alpha=\varepsilon_{r_{1}} \ldots \varepsilon_{r_{n}}[s]
$$

and

$$
\beta=\varepsilon_{t_{1}} \ldots \varepsilon_{t_{m}}[u]
$$

are in standard form and that $B^{\alpha}=B^{\beta}$. Then, because of (5.4.ii), that is, because $B^{\alpha} \subseteq B_{s}$ and $B^{\beta} \subseteq B_{u}$, we must have $s=u$.

On the other hand, $B^{\alpha} B^{\alpha^{*}}=B^{\alpha \alpha^{*}}$, but $\alpha \alpha^{*}=\varepsilon_{r_{1}} \ldots \varepsilon_{r_{n}} \varepsilon_{s}$. So, by our claim, we have $B^{\alpha \alpha^{*}}=\overline{\operatorname{span}}\left\{P_{E} \delta_{e}: e, r_{1}, \ldots, r_{n}, s \in E\right\}$, which is a subspace of $B_{e}$. Since 
$B^{\alpha}=B^{\beta}$, this subspace must coincide with

$$
B^{\beta \beta^{*}}=\overline{\operatorname{span}}\left\{P_{E} \delta_{e}: e, t_{1}, \ldots, t_{m}, u \in E\right\}
$$

from which the final conclusion follows easily.

In the special case of finite groups we have therefore proven:

6.8. Corollary. Let $G$ be a finite group of order $p$. Then, there is a G-graded $C^{*}$-algebra $A=\bigoplus_{t \in G} A_{t}$, such that the subsemigroup of $\mathcal{P}_{\ell}(A)$ generated by the grading spaces has exactly $2^{p-2}(p+1)$ elements; while, for any $G$-graded $C^{*}$-algebra the said subsemigroup cannot have more than this number of elements.

Beyond the purpose of constructing interesting graded $C^{*}$-algebras, we believe there is a considerable amount of interesting information that can be dug out from the structure of $C_{p}^{*}(G)$.

To deal with the specific case of finite groups, one can prove that $C_{p}^{*}(G)$ is also the groupoid $C^{*}$-algebra [13] constructed from the partial action of $G$ on $C(K)$, and compute $C_{p}^{*}(G)$ for the most elementary finite groups. In particular, for the two groups of order 4 , namely $Z / 4 Z$ and $Z / 2 Z \oplus Z / 2 Z$, one obtains

$$
C_{p}^{*}(Z / 4 Z)=C^{7} \oplus M_{2}(C) \oplus M_{3}(C)
$$

while

$$
C_{p}^{*}(Z / 2 Z \oplus Z / 2 Z)=C^{11} \oplus M_{3}(C)
$$

It is quite intriguing that, while the group $C^{*}$-algebra of finite commutative groups forgets everything but the order of the group, the partial group $C^{*}$-algebra $C_{p}^{*}(G)$ allows us to distinguish between $Z / 4 Z$ and $Z / 2 Z \oplus Z / 2 Z$.

\section{REFERENCES}

1. B. Abadie, S. Eilers and R. Exel, "Morita equivalence and crossed products by Hilbert $C^{*}-$ bimodules", preprint, Universidade de São Paulo, 1994, to appear in Trans. Amer. Math. Soc. CMP 97:17

2. B. A. Barnes, Representations of the $l_{1}$ algebra of an inverse semigroup, Trans. Amer. Math. Soc. 218, (1976), 361-396. MR 53:1169

3. J. Duncan and A. L. T. Paterson, " $C^{*}$-algebras of inverse semigroups", Proc. Edinburgh Math. Soc. 28 (1985), 41-58. MR 86h:46090

4. R. Exel, "Twisted Partial Actions, A Classification of Regular $C^{*}$-Algebraic Bundles", Proc. London Math. Soc. 74 (1997), 417-443. CMP 97:05

5. R. Exel, "Circle Actions on $C^{*}$-Algebras, Partial Automorphisms and a Generalized PimsnerVoiculescu Exact Sequence", J. Funct. Analysis 122 (1994), 361-401. MR 95g:46122

6. J. M. G. Fell and R. S. Doran, "Representations of *-algebras, locally compact groups, and Banach *-algebraic bundles", Pure and Applied Mathematics. vol. 125 and 126, Academic Press, 1988. MR 90c:46001; MR 90c: 46002

7. J. M. Howie, "An introduction to Semigroup theory", Academic Press, 1976. MR 57:6235

8. K. Jensen and K. Thomsen, "Elements of KK-Theory", Birkhäuser, 1991. MR 94b:19008

9. K. McClanahan, " $K$-theory for partial crossed products by discrete groups", J. Funct. Analysis, 130 (1995), 77-117. MR 95i:46083

10. G. K. Pedersen, " $C^{*}$-Algebras and their automorphism groups", Acad. Press, 1979. MR 81e: 46037

11. M. Pimsner, "A class of $C^{*}$-algebras generalizing both Cuntz-Krieger algebras and crossed products by Z", Amer. Math. Soc., Providence, R.I., 1997. MR 97k:46069 
12. J. Quigg and I. Raeburn, "Landstad duality for partial actions", preprint, University of Newcastle, 1994.

13. J. Renault, "A groupoid approach to $C^{*}$-algebras", Lecture Notes in Mathematics, vol. 793, Springer, 1980. MR 82h:46075

14. N. Sieben, " $C^{*}$-crossed products by partial actions and actions of inverse semigroups", Masters Thesis, Arizona State University, 1994. CMP 97:14

Departamento de Matemática, Universidade de São Paulo, Rua do Matão, 1010, 05508-900 São Paulo, Brazil

Current address: Departamento de Matemática, Universidade Federal de Santa Catarina, 88010-970 Florianópolis, SC, Brazil

E-mail address: exel@mtm.ufsc.br 